\title{
Our Exciting Journey to ACT-451840
}

\author{
Christoph Boss ${ }^{\star a}$ and Sergio Wittlin*bc
}

\begin{abstract}
We describe our work resulting in the selection of ACT-451840 (38) as a novel antimalarial drug with a novel mode of action. The compound was broadly characterized in vitro as well as in vivo in rat PK experiments as well as two different mouse malaria models. In the $P$. berghei infected mouse model cure could be achieved at oral doses of $300 \mathrm{mg} / \mathrm{kg}$ over 3 consecutive days. ACT-451840 was clinically investigated up to an experimental human malaria infection model, where therapeutic effects could be shown.
\end{abstract}

Keywords: Academia-Industry collaboration · ACT-451840 - Drug discovery · Malaria $\cdot$ Medicinal chemistry

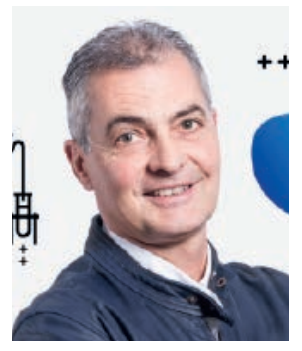

Christoph Boss completed his $\mathrm{PhD}$ in syn+ thetic organic chemistry at the University of Bern (Prof. Reinhart Keese), Switzerland followed by post-doctoral training at The Scripps Research Institute (TSRI; Prof. Julius Rebek, Jr.) in LaJolla, California, USA. From 1999 until 2012, he had various positions of increasing responsibility in Actelion's drug discovery chemistry department contributing to the identification of several lead compounds including macitentan (Opsumit) for PAH and daridorexant for insomnia. From November 2012 to June 2017, he was the Senior Group Leader Chemistry Technologies at Actelion Pharmaceuticals Ltd. He joined Idorsia when the company was established in June 2017 as the Senior Group Leader in Drug Discovery Chemistry, Oncology. He was appointed Head of Drug Discovery Chemistry and member of the Idorsia leadership team in July 2019. In 2019, Christoph Boss received the SCS Industrial Science Award from the Swiss Chemical Society.

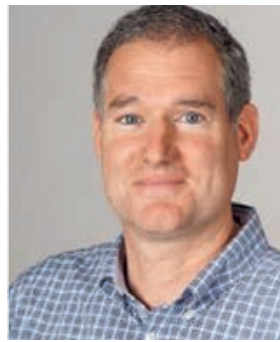

Sergio Wittlin is a group leader at the Swiss Tropical and Public Health Institute (Swiss $\mathrm{TPH})$. He received his $\mathrm{PhD}$ in biochemistry from the Biozentrum (University of Basel, Switzerland) in 1999 and obtained three years of postdoctoral experience in molecular genetics at the Walter and Eliza Hall Institute (Melbourne, Australia). In 2002 he moved to the Swiss TPH, where his research is focused on the malaria parasite in cell culture assays and mouse models, with the ultimate aim to discover new antimalarial drugs in a multidisciplinary approach. In 15 years of collaboration with the Medicines for Malaria Venture (MMV) in Geneva, his laboratory was significantly involved in moving five compounds in the MMV pipeline into clinical trials. In 2021, Sergio Wittlin successfully completed his habilitation at the University of Basel.

${ }^{*}$ Correspondence: Dr. C. Boss ${ }^{\mathrm{a}}$, Dr. S. Wittlin ${ }^{\mathrm{bc}}$

E-mail: christoph.boss@idorsia.com, sergio.wittlin@swisstph.ch

aDrug Discovery and Preclinical Development, Idorsia Pharmaceuticals Ltd,

Hegenheimermattweg 91, CH-4123 Allschwil, Switzerland;

bSwiss Tropical and Public Health Institute,

Socinstrasse 57, CH-4002 Basel, Switzerland;

\section{Malaria}

Malaria is a life-threatening disease which is caused by parasites. The parasites are transmitted to humans by the bites of infected female Anopheles mosquitos. The disease can be prevented and cured by medication. There were an estimated 229 million malaria cases worldwide in 2019 which resulted in an estimated 409,000 deaths. Children under the age of 5 years are the most vulnerable population affected by malaria. In 2019 they accounted for 274,000 fatal cases, reflecting $67 \%$ of all malaria deaths. The most strongly affected region is the WHO African region with $94 \%$ of the malaria cases and deaths in 2019. Funding for malaria control and elimination was approximately 3 billion USD in 2019 with 900 million USD coming from governments of endemic countries.

Malaria remains a leading cause of death with an important impact on public health and economic aspects in the poor areas of the world. The recent increases in cases is due to reduced efforts to combat malaria but also due to emerging resistance to the artemisinin-based combination therapy (ACTs), manifesting itself as slower rates of parasite clearance in treated patients, and the absence of efficacious vaccines or the very limited chemotherapeutic alternatives to the ACTs. ${ }^{[1]}$

\subsection{Historic Context}

The first Global Malaria Eradication Programme ended in 1969. This resulted in a reduction of political commitment and funding for efforts to control and eradicate malaria and obviously led to a recurrence of malaria on large scale, especially in Africa with hundreds of millions of people likely being infected and tens of millions dying. In the early 1990s, a new plan to fight malaria was developed by senior health leaders together with scientists. As a consequence, more funding became available for research, driving innovation in diverse areas such as insecticide-treated nets, improved and rapid diagnostic tests and novel, more effective treatments. New financing mechanisms such as the Global Fund to Fight AIDS, Tuberculosis and Malaria and the US President's Malaria Initiative, allowed for a wide distribution of the novel tools to fight malaria and most significantly contributed to an important reduction in infections and death cases. Strong political commitment by African leaders was of key importance to success. The goal of the African leaders, formulated in 2000 in the Abujy Declaration was to reduce malaria mortality by $50 \%$ over 10 years. Based on the WHO Malaria report, malaria mortality was reduced by $60 \%$ from 2000 to 2019 . The African region achieved strong reductions in death cases from 680,000 in 2000 down to 384,000 in 2019. The countries 
in South-East Asia also experienced a strong decrease in cases and deaths of $73 \%$ and $74 \%$, respectively. In India alone the cases went down from estimated 20 million to approximately 6 million. Over the last 20 years, twenty-one countries have eliminated malaria. Ten out of the twenty-one countries were certified malaria free by the WHO. Improvements in the situation with respect to malaria were very impressive over the last two decades. But the 2020 Malaria report of the WHO[1] clearly confirms the trend observed over the past years, that progress has slowed down significantly. Already in 2017, the WHO warned that the global response to malaria was at a level that the goals of the WHO's global malaria strategy will be missed with high probability. In 2020 the progress is plateauing. The 2020 targets for reductions in infections and death were missed by $37 \%$ and $22 \%$, respectively. In the spring of 2020 COVID-19 appeared as an additional huge challenge and negatively impacted the malaria response all over the world, making it more difficult and dangerous for health workers to provide the services to the malaria affected population. The WHO report estimates that a $25 \%$ disruption to antimalarial treatments in Sub-Saharan Africa could result in 46,000 additional deaths. To reanimate efforts to combat malaria, the WHO helped set-up the 'high burden to high impact' (HBHI) program in 2018, in collaboration with the Roll Back Malaria initiative. The HBHI program is run with a focus on 11 countries, 10 of which are located in Sub-Saharan Africa, facing approximately $70 \%$ of the world's malaria infections. HBHI countries develop individual, tailored approaches for malaria control to replace the standard one-size fits all strategy. The hope is, that by specific measures in high burden countries the impact which can be reached with the available means can be maximized for the whole region and also the world. A better targeting of interventions and resources in high burden areas will help to increase the speed in progress and innovation and come up with better tools and medications. In addition, efforts to fight diseases like malaria, should be integrated into efforts to build strong health systems to maximize sustainability. The situation as of 2020 clearly indicates how crucial all these initiatives are for the goal to eradicate malaria within a reasonable timeframe.

\subsection{Today's Treatment Options}

A perfect antimalarial drug would allow for the following four clinical outcomes: prevent the infection, treat the symptoms, prevent relapses, and block transmission. Today, different drugs are administered to obtain the four effects:[2]

- Chemoprevention is most efficiently achieved by mefloquine (Lariam ${ }^{\circledR}$, Fig. 1). In addition, the combination of atovaquone and proguanil (Malarone ${ }^{\circledR}$ ) is used. Fansidar ${ }^{\circledR}$, a combination of sulfadoxin/pyrimethamine is used preventively in pregnant women and children due to its excellent safety profile. This treatment option unfortunately is losing efficacy due to newly developed resistances.

- Uncomplicated malaria is treated with ACTs. Artemisinin and its derivatives show a fast onset of action and are cleared rapidly $\left(\mathrm{t}_{1 / 2}=1 \mathrm{~h}\right)$. They need to be combined with an agent having a long $t_{1 / 2}$ to eliminate remaining parasites. Usually the combinations are administered for 3 days. The best known are $\operatorname{Coartem}^{\circledR}$, a combination of artemether and lumefantrine or Eurartesim ${ }^{\circledR}$ consisting of dihydroartemisinin and piperaquine. Treatment of life-threatening malaria is usually achieved by parenteral administration of artesunate.

- To prevent relapses a 14-day treatment of primaquine is prescribed. This drug exhibits an elevated risk of hemolytic anemia in patients with glucose-6-phosphatedehydrogenase (G6PD) deficiency ( $10 \%$ of the population). Testing for G6DP deficiency is necessary before treatment. The same counts for the recently approved tafenoquine, which has the advantage that it has to be dosed less frequently. Tafenoquine and primaquine are the only available drugs which efficiently block malaria transmission.

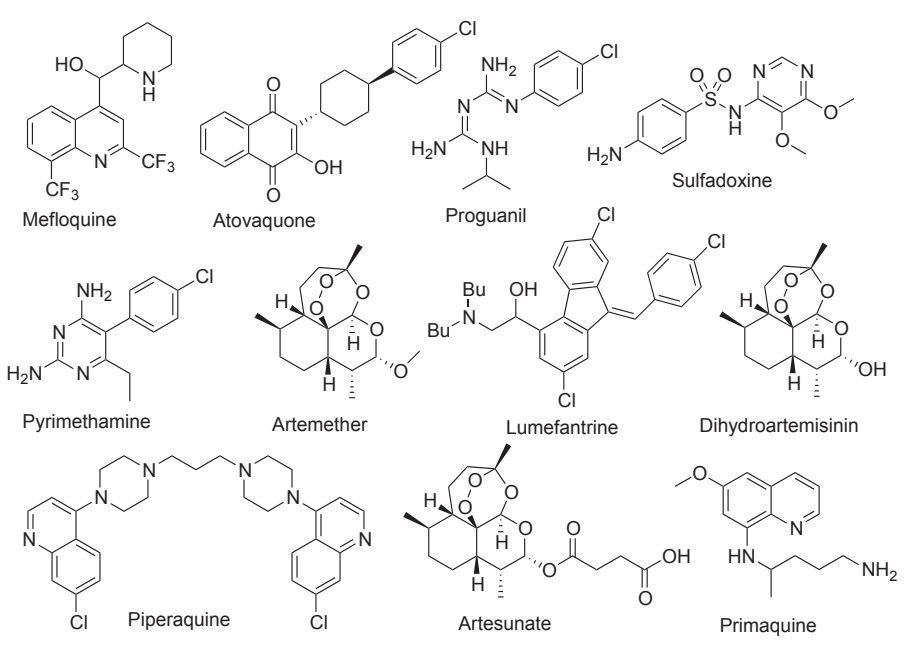

Fig. 1. Chemical structures of antimalarials in current use.

Even though significant progress in malaria research has been made over the last two decades, the ideal drug has not yet materialized. The situation is complicated by the fact that malaria is caused by five different parasite species, Plasmodium falciparum, Plasmodium vivax, Plasmodium malariae, Plasmodium ovale and Plasmodium knowlesi, each exhibiting a partially different biology. The life cycle of the malaria parasites is complex and divided into different stages presenting an additional obstacle to intervene in the natural course of infection. When a malaria parasite infects a human, the Plasmodium first enters the liver, therefore targeting the liver is key for prevention. $P$. vivax and $P$. ovale are able to remain dormant in the liver (as hypnozoites) and can cause relapses of malaria even after many years. To prevent relapses, a drug must eliminate hypnozoites. From the liver, parasites migrate to erythrocytes in the blood. Rapid proliferation leads to the symptoms of malaria. To treat the symptoms, a drug must attack the blood stage of the parasites. Finally, some of the parasites develop into gametocytes. When gametocytes are taken up by a mosquito during a blood meal, they develop into new parasites within the mosquito, ready to infect the next victim. Killing the gametocytes could block transmission of malaria. Due to the complex biology and the complicated parasite life cycle the hurdle for one single agent to achieve all the tasks simultaneously is very high. As of today, novel treatment regimens therefore will be combinations of two or more components whose properties must be optimised for the specific purpose. For example, treatment of pregnant women and children requires highest safety standards. As malaria is predominantly occurring in poor areas of the world, where co-infections, often with HIV are common, drug-drug interaction potential has to be closely monitored. In addition, the molecules need to be chemically stable, easy to handle and cost of goods must be cheap.

\section{The Collaboration - How did it start}

At the beginning of Actelion in 1999 the founders decided to focus on two families of targets, representing the two legs the research organisation should stand on. The first leg was G-ProteinCoupled Receptors, where drugs such as Macitentan (Opsumit ${ }^{\circledR}$ ), Ponesimod (Ponvory ${ }^{\circledR}$ ) or Daridorexant (NDA filed) were identified. The second leg was aspartic proteases as targets. The most interesting aspartic protease targets at that time in the industry were HIV protease or renin, then beta-secretase (BACE). We were very interested in renin as a cardiovascular target. In order to generate experience with the target class and gain experience in 
technology related to expression, purification and crystallisation of aspartic proteases, we started to work on plasmepsin II (PMII), an aspartic protease found in the malaria parasite. We worked on this target educating master students from different universities in Switzerland, France and Germany, in the medicinal and highthroughput chemistry labs. Once interesting inhibitors of PMII were identified, we contacted the Swiss TPH to investigate our PMII inhibitors in their red blood cell (RBC) based phenotypic malaria parasite in vitro assays. The experience was quite sobering. Our enzyme inhibitory activity never really translated into antiparasitic activity in the RBC assay. We and others learned that inhibiting PMII in presence of several other plasmepsins in the parasite did not harm the parasite. It became obvious that one would have to inhibit multiple plasmepsins to kill the parasite. We therefore stopped this project but decided to continue our collaboration with the Swiss TPH in the field of antimalarials and started the project again in a completely different manner.

\subsection{The Industrial Perspective - The Academic Perspective}

In this collaboration, we from the industry side (Actelion Pharmaceuticals at the time, Idorsia Pharmaceuticals now) and we from the academic side (Swiss TPH, University of Basel) made the following key learnings:

- In the extreme specialist field of malaria research, the academic specialists are key in developing an understanding of the biology, the mode of action investigations, the target identification efforts, the in-depth characterization of the drug in preclinical development (activities on the different parasite stages, comparative data generation with marketed antimalarials, characterization of the drug in humanized mouse models, resistance development frequency assessment).

- With respect to medicinal chemistry optimization and DMPK characterization of potential drug candidates, the industrial partner are the specialists. The infrastructure at the university in these areas is not comparable and not competitive to what industry can offer.

- All aspects of production of drug substance and drug product have to be led and guided by the industry partner.

- Phase I and Phase II clinical development activities were driven by the industry partner.

- Switzerland has a long-standng commitment to international cooperation and hosts, e.g. the WHO since 1946, the Red Cross since 1863 and more recent organizations such as the Medicines for Malaria Venture (MMV, since 1999) or the Drugs for Neglected Diseases Network (DNDi, since 2003). Swiss TPH is used to collaborate with those organizations since its foundation in 1943 and provides as such a solid network to advance drugs in the field of malaria, for example. ${ }^{[3]}$

- A collaboration can only be effective if all IP aspects are agreed on early. The situation is easy in fields where not much profit can be envisaged.

\subsection{The New Attempt}

We decided to perform a screen of 5000 randomly selected compounds from the Actelion screening compound collection in an erythrocyte-based phenotypic assay against the chloroquineresistant K1 strain of Plasmodium falciparum at the Swiss TPH. We identified several hits, among them the phenylalanine based compound $\mathbf{1}$ (Fig. 2). Compound $\mathbf{1}$ is very attractive as a starting point for lead optimization as it already exhibited significant activity (IC50 K1 alb $=3.8 \mathrm{nM}$ ) in the RBC assay. In addition, it offers immediately seven areas to be investigated during lead optimization.

The influence of the chirality of the amino acid needs to be clarified (A). The active enantiomer exhibiting the chirality of the natural amino acid would be an advantage, certainly for the cost of goods. The question if the phenylalanine core can be replaced by other amino acids (a)) needs clarification and if the phenylalanine remains, investigation of potential substitution patterns on the phenyl ring will have to be done. The piperazine moiety (B) immediately triggers ideas for variations (e.g. ring size; open chain analogues) and the benzyl amine unit (b)) asks for investigation of heteroaromatic replacements and substitution patterns at the aromatic ring and to check if the basic nature of the piperazine $\mathrm{N}$-atom is required or if amide formation or lactam formation is tolerated. The pentyl-benzyl moiety (c)) inspires many ideas for replacements, certainly the pentyl chain should immediately be replaced by more drug-like substituents. The cinnamic acid moiety $(\mathbf{C})$ raises the question of the importance of the $\mathrm{C}=\mathrm{C}$ double bond for activity and with respect to safety, the potential reactivity as a Michael acceptor needs to be clarified. It also has to be investigated if the phenyl ring (d)) can be replaced by heteroaromatic systems as well as the substitution pattern has to be clarified.

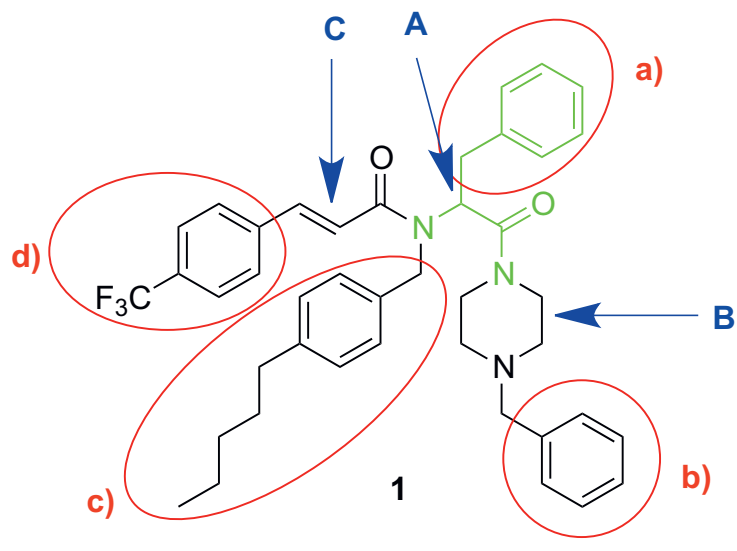

Fig. 2. Phenylalanine based screening hit from phenotypic RBC assay

An important aspect in antimalaria drug discovery is the cost of goods. As can be seen in Fig. 3, ${ }^{[4]}$ the chemical accessibility of compound $\mathbf{1}$ and its derivatives is simple and straightforward. This is an important advantage. The synthesis was started by coupling the Boc-protected phenylalanine (2) with benzylpiperazine (3) applying standard peptide bond forming conditions, followed by Boc-cleavage with TFA in dichloromethane to obtain compound 4. The use of $\mathrm{HCl}$ in dioxane to cleave the Boc group often resulted in the decomposition of the piperazinyl-amide. Derivative 4 was transformed into compound $\mathbf{6}$ in a reductive amination reaction with the substituted benzaldehyde $\mathbf{5}$ using dichloromethane or acetonitrile as a solvent and sodium tri-acetoxy borohydride as the reducing agent. In order to obtain the final compound $\mathbf{1}$, precursor 6 was reacted with a cinnamic acid derivative 7 in a simple acylation reaction. Details on the synthetic procedures can be found in the patent literature. ${ }^{[5]}$ Following this general strategy (sometimes changing the order of the steps), we prepared approximately 5000 compounds by students during their master thesis, over about seven years.

The final compounds were tested for inhibition of parasite growth in RBCs infected with the P. falciparum parasite strain $\mathrm{K} 1$ (chloroquine resistant) and/or with the chloroquine-sensitive strain NF54. Our compounds were active against chloroquine-resistant as well as -sensitive strains, as both assays produced comparable results. Parasites were cultured in vitro according to Trager and Jensen. ${ }^{[6]}$ IC50 values were determined by measuring incorporation of the nucleic acid precursor $[3 \mathrm{H}]$ hypoxanthine after 72 hours of incubation. ${ }^{[7,8]}$ IC50 values were usually determined in the presence of $0.5 \%$ Albumax (a serum substitute corresponding to a final assay concentration of $10 \%$ bovine serum albumin). 

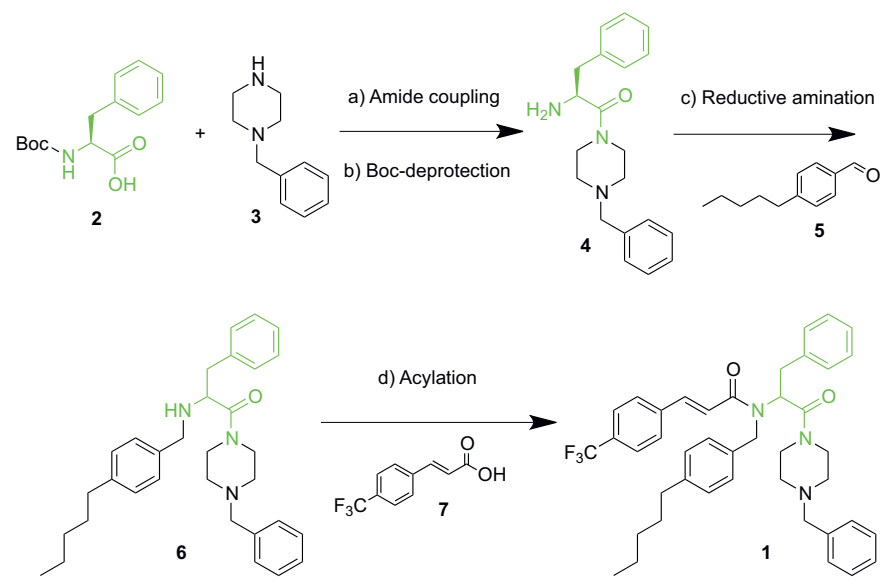

Fig. 3. Synthesis of screening hit 1 to illustrate the general accessibility of the phenyl-glycine based derivatives.

Depicted in Fig. 4 is the small cluster of compounds, with closely related chemical structures, initially prepared for a completely different project, which were all identified as hits in the phenotypic antimalarial screen. This added confidence to the results and gave first insights into the Structure-Activity Relationship (SAR). Varying the position of the substituent of the phenyl ring of the cinnamoyl moiety, pointed out that substitution of the para position resulted in more active compounds $(\mathbf{1 , 1 0})$ as compared to the meta-substituted analogues $(\mathbf{8}, \mathbf{1 1})$ and the ortho-substituted derivative 9.

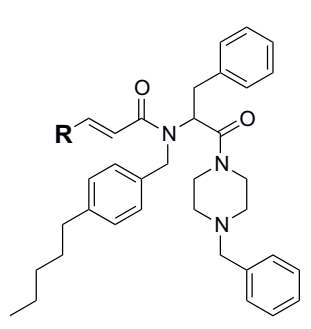

Cmpd
1
8
9
9

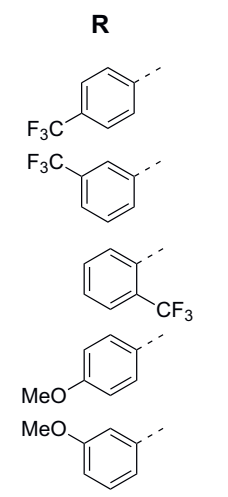

$\mathrm{IC}_{50} \mathrm{~K} 1 \mathrm{alb}[\mathrm{nM}]$

3.8

Fig. 4. Hit cluster with initial SAR on cinnamic acid substitution.

A question of key importance was the influence of the chirality of the phenylalanine core on antimalarial activity. In view of cost of goods, a necessity of unnatural amino acid chirality for antimalarial activity could be an immediate showstopper.

The two compounds $\mathbf{1 2}$ and 13, depicted in Fig. 5 differ only by the chirality of the phenylalanine core and indicate that the naturally occurring $S$-configuration present in $\mathbf{1 2}$ results in the compound exhibiting significantly higher antimalarial activity as compared to $\mathbf{1 3}$ with the unnatural $R$-configuration present in the amino acid core, being almost inactive.

Next questions we wanted to answer were the importance of the $\mathrm{C}=\mathrm{C}$ double bond, the length of the linker between the aryl moiety and the N-terminus of the core phenylalanine and also the importance of the aryl moiety for antimalarial activity. A few relevant datapoints are summarized in Fig. 6.

In the examples given in Fig. 6 we also replaced the originally present n-pentyl substituent with a pyridine ring as a step toward better molecular properties. Substantial activity can be retained by replacing the $\mathrm{C}=\mathrm{C}$-double bond with a single bond as can be seen in 14. The addition of a methylene moiety to a C3-linker resulted

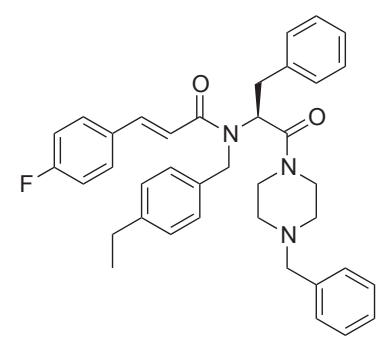

12: S-chirality, natural AA $\mathrm{IC}_{50} \mathrm{~K} 1 \mathrm{alb}: 13 \mathrm{nM}$

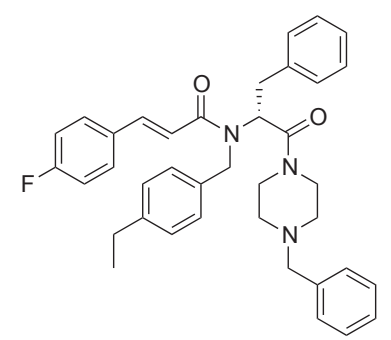

13: R-chirality, non-natural $A A$ $\mathrm{IC}_{50} \mathrm{~K} 1 \mathrm{alb}:>100 \mathrm{nM}$
Fig. 5. Impact of the chirality of the amino acid core on inhibitory activity.<smiles>[R]N(Cc1ccc(-c2ccncc2)cc1)[C@@H](Cc1ccccc1)C(=O)N1CCN(Cc2ccccc2)CC1</smiles>

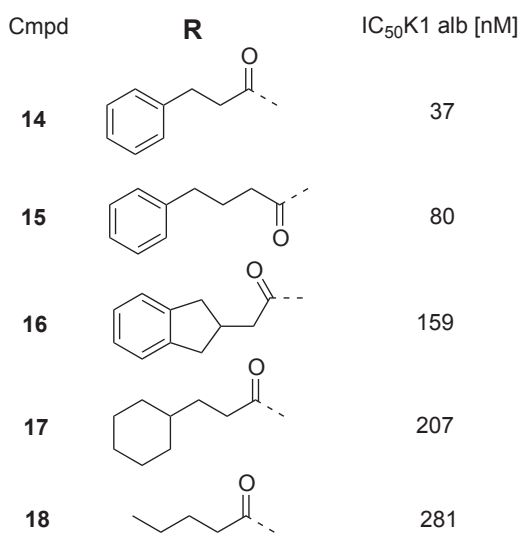

Fig. 6. Removal of the cinnamic acid $\mathrm{C}=\mathrm{C}$-double bond.

in a loss of activity (15). The indane system, used as a rigidified C3-linked analogue (16) resulted in a further loss of activity. Replacing the phenyl ring by a cyclohexyl ring (17) or complete removal of the phenyl ring and leaving a butyl chain (18) both had negative consequences on antimalarial activity. This investigation pointed out that we needed an aromatic ring system linked by a C2-linker via an amide bond to the amino acid core to obtain highly active compounds. We can play with hydro cinnamoyl and cinnamoyl moieties in this part of the molecules.

Next, we describe a summary of replacements we investigated for the n-pentyl-benzyl moiety present in the initial hit cluster depicted in Fig. 4 and keeping the rest of the molecules constant as found in compound $\mathbf{1}$ except that we worked with S-chirality of the amino acid core and avoided racemates. A selection of results is given in Fig. 7. In these investigations, we realized that our screening assay, using Albumax as culture medium supplement, suffered from limitations. Binding of our compounds to serum components reduced the free compound concentration in the assay and resulted in an overestimation of the compound's potency in the absence of serum components. The assay environment was therefore optimized by adding $50 \%$ of human serum (final assay concentration) to the media. Under these conditions, the relevance of the physicochemical properties of our compounds for antimalarial activity was significantly increased. The results became more meaningful 
for subsequent planning of in vivo experiments based on in vitro results. In antimalarial drug discovery, in vivo experiments are usually performed in mice infected by the naturally occurring rodent malaria parasite $P$. berghei and not by $P$. falciparum, which infects only humans. Therefore, we developed an in vitro assay to determine the activity of our compounds toward the rodent malaria parasite $P$. berghei. This assay was done in Albumax-containing media and had an incubation time of $24 \mathrm{~h}$. We learned that our antimalarials were less effective against the murine $P$. berghei parasites than against the human parasite strains. This compelled us to work with a humanized $P$. falciparum SCID mouse model to generate relevant in vivo assessments of our best compounds.

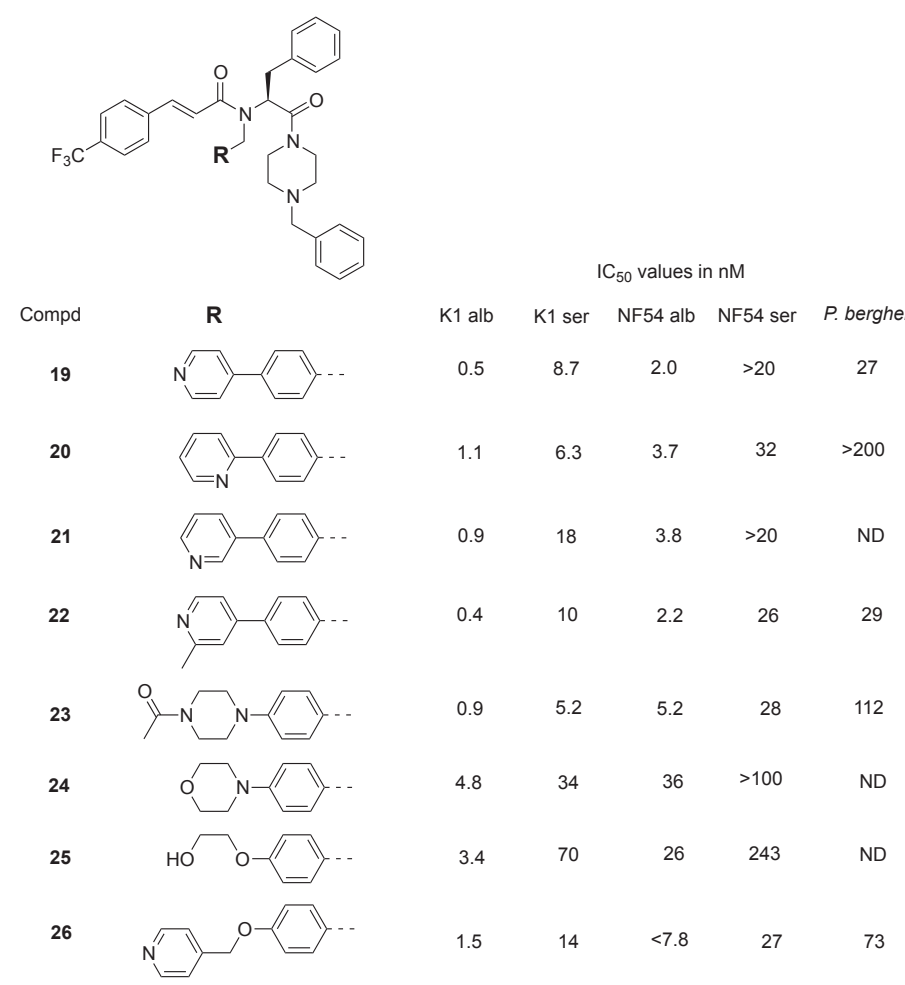

Fig. 7. Replacement of the n-pentyl-benzoyl moiety.

In general, all compounds depicted in Fig. 7 exhibited significant antimalarial activity under Albumax conditions, against the K1 as well as against the NF54 parasite strain. Moving toward the serum assay conditions, for each parasite strain a loss of activity was observed. As discussed, the activity of our antimalarials against the murine $P$. berghei strain was reduced, which is confirmed with all compounds summarized in Fig. 7. This was a rather surprising finding, as most of the modern antimalarials exhibit similar potency against the human and rodent parasites.

The data given in Fig. 7 were interpreted taking the diverse assumptions explained above into account. We did not only want to see low absolute antimalarial potency but also low shift factors between the assay conditions.

Taking this into account, compound $\mathbf{2 3}$ was the most interesting compound for us exhibiting shift factors of 5 to 6 between the Albumax and the human serum conditions, combined with very good antimalarial potency. We started to check the compounds for their cytochrome inhibition. Compound $\mathbf{2 3}$ resulted in an unproblematic CYP3A4 inhibition of $8.4 \mu \mathrm{M}$ with testosterone as a marker substrate, as compared to e.g. 19 exhibiting sub-micro molar potency in the CYP3A4 inhibition assay, due to the 4-pyridyl moiety present in $\mathbf{1 9}$. This could be prevented by introducing a substituent next to the pyridyl-N-atom as shown in 22, resulting in an improved CYP3A4 inhibition value of $5.3 \mu \mathrm{M}$. For further investigations we therefore often focused on the 4-N-acetylpiperazine-substituted benzyl group as present in 23.

We clarified the question of the optimal substitution position of the benzyl ring by preparing a few meta- as well as ortho-arranged bis-aryl and related systems (results not depicted here). This investigation confirmed that the para-arrangement always resulted in significantly more active derivatives. In addition, a broad investigation on the substitution pattern of the aryl and heteroaryl moiety of the cinnamic acid was performed and the detailed results were published elsewhere. ${ }^{[4]}$ Most interesting results were obtained with a trifluoromethyl, a t-butyl or an iso-propoxy group in para-position.

We also did extensive SAR work on the phenylalanine core by replacing the phenyl ring with heteroaromatic systems such as different pyridyl, pyrimidinyl, pyridazinyl isomers or 5-membered heteroaromatic groups such as pyrrolyl, pyrazolyl, imidazolyl or isoxazolyl as well as saturated heterocycles such as piperidinyl, morpholinyl or piperazinyl. None of these replacements resulted in significant advantages and were therefore not further pursued. In Fig. 8 (compounds 27 to 29) the results of a fluorine scan at the phenyl ring of the phenylalanine core are summarized. No advantage of additional substitution on the amino acid core phenyl ring was identified. The natural phenylalanine still resulted in the best compounds. This clearly is an advantage with respect to cost of goods.

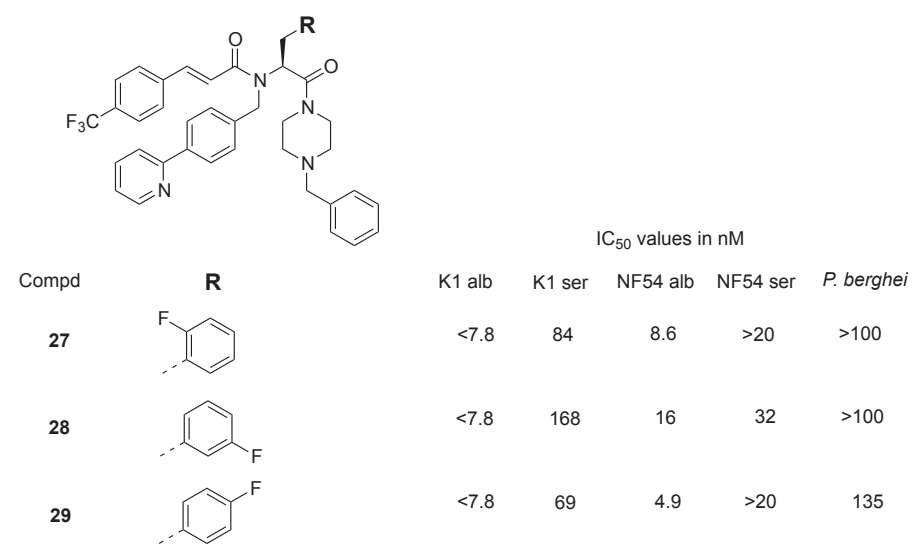

Fig. 8. Fluorophenylalanine derivatives.

Fig. 9 depicts a representative summary of the variations performed at the benzyl-piperazine part of the initial hit. Compounds $\mathbf{3 0}$ and $\mathbf{3 1}$ revealed that an electron-withdrawing substituent in the para-position of the phenyl ring had a positive effect on antimalarial activity, which disappeared when the nitrile substituent was put in meta-position. Compound 32, bearing a chlorine atom in para-position resulted in lower activity and a tendency for a higher shift between the Albumax and the serum conditions, especially in the NF54 assay. Compound $\mathbf{3 3}$ showed that polar substituents are tolerated in this area. We further profiled this compound but it turned out to be less convincing compared to ACT-451840. ${ }^{[4]}$ The benzoyl derivative $\mathbf{3 4}$ was significantly less potent as compared to the basic amine analogues, especially under human serum containing assay conditions. Acylation was not further pursued. Replacing the phenyl ring with small alkyl units with a polar head group $(\mathbf{3 5}, \mathbf{3 6})$ resulted in less active antimalarials as well as increasing the linker length by an additional methylene group as depicted for compound $\mathbf{3 7}$.

Further optimization work by combining identified substituents leading to attractive activities and properties finally resulted in the selection of $\mathbf{3 8}$ (ACT-451840) as the compound we wanted to further characterize and define if it qualified as a clinical development candidate (Fig. 10). 


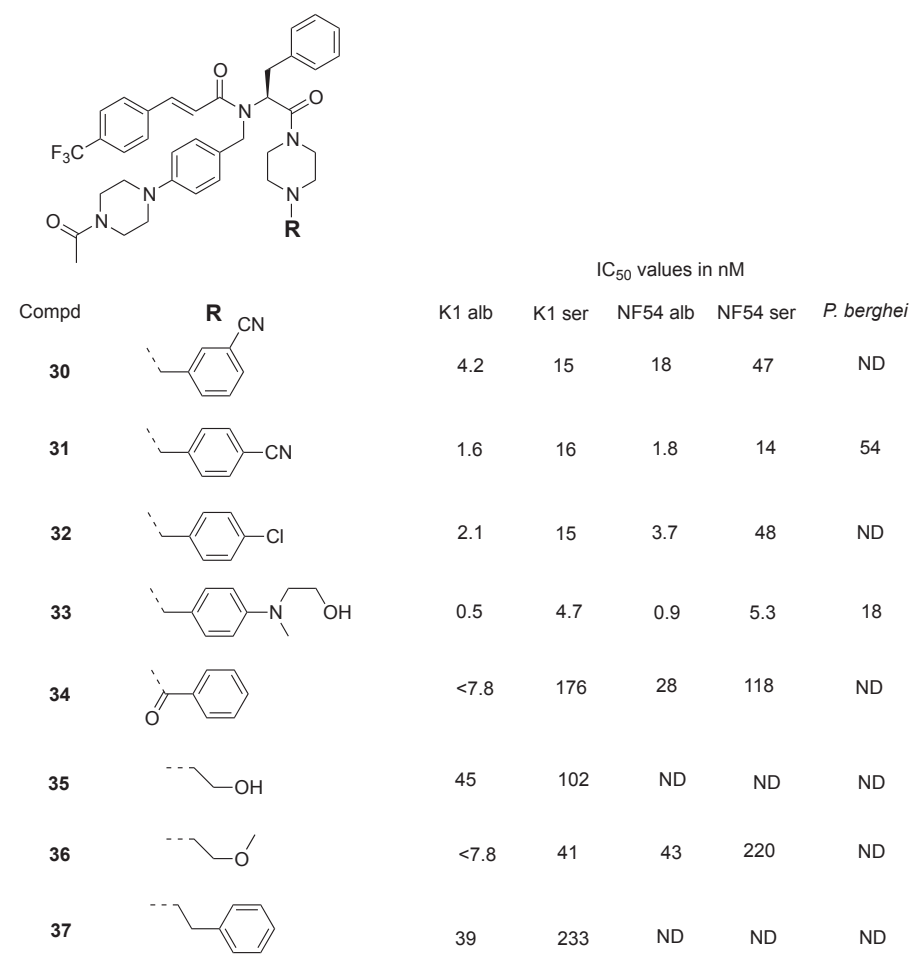

Fig. 9. SAR on the benzylpiperazine part.

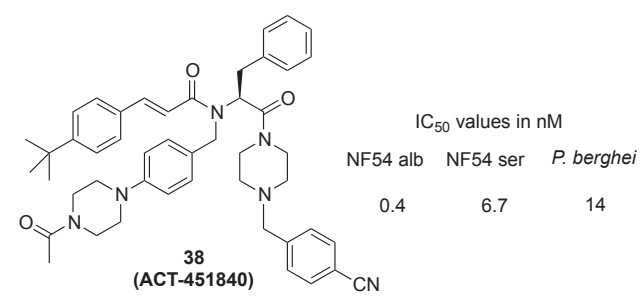

Fig. 10. In vitro data for compound 38 (ACT-451840). Selected DMPK parameters (in vivo data generated in rats; $10 \mathrm{mg} / \mathrm{kg}$ p.o. and $1 \mathrm{mg} / \mathrm{kg}$ i.v.); $\mathrm{CL}=57 \mathrm{~mL} /\left(\mathrm{min}^{*} \mathrm{~kg}\right) ; \mathrm{Vss}=7.6 \mathrm{~L} / \mathrm{kg} ; \mathrm{t} 1 / 2=2.3 \mathrm{~h} ; \mathrm{AUCp} .0 .=1270$ $\mathrm{ng}^{*} \mathrm{~h} / \mathrm{mL} ;$ AUCi.v. $=291 \mathrm{nf}{ }^{*} \mathrm{~h} / \mathrm{mL} ; \mathrm{Cmax}=288 \mathrm{ng} / \mathrm{mL} ; \mathrm{tmax}=0.5 \mathrm{~h} ; \mathrm{F}$ $=44 \% ; \mathrm{HLM}$ CLint $=267 \mathrm{~mL} /\left(\mathrm{min}^{*} \mathrm{~kg}\right) ; \mathrm{RLM}$ CLint $=111 \mathrm{~mL} /\left(\mathrm{min}^{*} \mathrm{~kg}\right)$; $\mathrm{hPPB}=99.9 ; \mathrm{rPPB}=99.9$.

\section{DMPK Assessment of ACT-451840 (Compound 38)}

With respect to in vitro antimalarial activity, $\mathbf{3 8}$ showed excellent potency against the CQ sensitive NF54 parasite strain with 0.4 $\mathrm{nM}$ under albumax conditions and $6.7 \mathrm{nM}$ in the presence of $50 \%$ serum. We selected interesting compounds based on the NF54 assay data for technical reasons. This assay resulted in more relevant anti-malarial activity as compared to the $\mathrm{K} 1$ assay, where our compounds usually behaved more efficacious. Also 38, as seen with previous compounds, showed a reduced potency towards the mouse malaria strain $P$. berghei. In the human liver microsomal (HLM) assay, the Clint value was in the same range as the value obtained in the rat liver microsomal (RLM) assay. In vitro-in vivo-estimation (IVIVE) would therefore predict higher clearance in humans as compared to rats. The half-life $\left(\mathrm{t}_{1 / 2}\right)$ after intravenous (i.v.) administration was $2.3 \mathrm{~h}$ which fits well with a medium to high clearance compound. The volume of distribution (Vss) is above total body water, indicating high tissue distribution and limited concentrations in the systemic compartment. This is consistent with the rather high molecular weight and the resulting lipophilic character of $\mathbf{3 8}$. Plasma protein binding (PPB) was high for humans as well as for rats. The oral bioavailability is often limited by high clearance. The rather high rat bioavailability of $44 \%$ for $\mathbf{3 8}$ was not completely understood. Our DMPK characterisation work in this project result- ed in the impression that working with compounds at the edge of the guideline of five, for example having more than one parameter above the given upper limits, increases difficulties in 'predicting' in vivo PK behaviour. ${ }^{[9]}$ Based on the pharmacology obtained with 38, we have shown that larger molecules may still have a potential of becoming drugs, but rational approaches are more difficult under these circumstances. We investigated $\mathbf{3 8}$ in in vivo pharmacology experiments under various dosing regimens in $P$. berghei infected mice. The results are summarized in Table 1 . The dose-escalating experiments with single doses from 10 to $60 \mathrm{mg} / \mathrm{kg}$ indicated that 38 started to exhibit significant anti-malarial effects (parasite reduction) in vivo at $20 \mathrm{mg} / \mathrm{kg}$. Repeated administration on three consecutive days resulted in curative activity at a dose of $300 \mathrm{mg} / \mathrm{kg}$ and significant antimalarial effects were seen already at $100 \mathrm{mg} /$ $\mathrm{kg}$. Compound 38 showed excellent reduction in parasitemia at $30 \mathrm{mg} / \mathrm{kg}$, resulting in $99.80 \%$ activity.

Table 1. In vivo antimalarial activity data for 38 (ACT-451840) in mouse experiments. $^{\text {a }}$

\begin{tabular}{|c|c|c|c|c|c|}
\hline $\begin{array}{c}\text { Dose } \\
{[\mathbf{m g} / \mathbf{k g}]}\end{array}$ & $\begin{array}{c}\text { Admini- } \\
\text { stration } \\
\text { Repeats }\end{array}$ & $\begin{array}{c}\text { Para- } \\
\text { sitized } \\
\text { RBC/100 }\end{array}$ & $\begin{array}{c}\text { \% of } \\
\text { Control }\end{array}$ & $\begin{array}{c}\text { Activity } \\
{[\%]}\end{array}$ & $\begin{array}{c}\text { Survival } \\
\text { days }\end{array}$ \\
\hline 100 & $1 \mathrm{x}^{\mathrm{b}}$ & 0.29 & 0.79 & 99.2 & 9.7 \\
\hline 60 & $1 \mathrm{x}^{\mathrm{c}}$ & 0.1 & 0.37 & 99.6 & 12.7 \\
\hline 30 & $1 \mathrm{x}^{\mathrm{c}}$ & 0.83 & 3.11 & 97 & 8.7 \\
\hline 25 & $1 \mathrm{x}^{\mathrm{c}}$ & 2.6 & 9.71 & 90 & 6.7 \\
\hline 20 & $1 \mathrm{x}^{\mathrm{c}}$ & 6.37 & 23.77 & 76 & 7.0 \\
\hline 15 & $1 \mathrm{x}^{\mathrm{c}}$ & 16.33 & 60.99 & $<40$ & 3.0 \\
\hline 10 & $1 \mathrm{x}^{\mathrm{c}}$ & 23.80 & 88.87 & $<40$ & 3.0 \\
\hline 300 & $3 \mathrm{x}^{\mathrm{cd}}$ & 0.17 & 0.39 & 99.6 & $30.0^{\mathrm{e}}$ \\
\hline 100 & $3 \mathrm{x}^{\mathrm{cd}}$ & 0.15 & 0.35 & 99.6 & 26.7 \\
\hline 30 & $3 \mathrm{x}^{\mathrm{cd}}$ & 0.10 & 0.23 & 99.8 & 13 \\
\hline 10 & $3 \mathrm{x}^{\mathrm{cd}}$ & 28.63 & 67.75 & $<40$ & 4.0 \\
\hline 3 & $3 x^{\mathrm{cd}}$ & 50.98 & 120.65 & $<40$ & 4.0 \\
\hline
\end{tabular}

\section{Preclinical and Clinical Results of ACT-451840 (Compound 38)}

Based on the data generated so far with $\mathbf{3 8}$ it was decided to select the compound for full preclinical development. ${ }^{[10]}$ Compound 38 showed excellent activity against several CQ-resistant as well as CQ-sensitive $P$. falciparum strains. It exhibited a low frequency of resistance development and a fast onset of action in vitro as well as in vivo as described above in the P. berghei mouse experiments and in a model of P. falciparum in humanized SCID mice at GSK in Tres Cantos, Spain. [11] Escalating oral dosing on four consecutive days showed a rapid onset of action and an effective dose resulting in $90 \%$ antimalarial activity $\left(\mathrm{ED}_{90}\right)$ of $3.7 \mathrm{mg} / \mathrm{kg}$, being similar to that of chloroquine after oral quadruple-dosing in this model $\left(\mathrm{ED}_{90}: 4.9 \mathrm{mg} / \mathrm{kg}\right)$. In the $P$. berghei infected mouse experiment, administration of $300 \mathrm{mg} / \mathrm{kg}$ of $\mathbf{3 8}$ on three consecutive days, resulted in cure and giving an $\mathrm{ED}_{90}$ value of $13 \mathrm{mg} / \mathrm{kg}$. The almost $4 \mathrm{x}$ difference in $\mathrm{ED}_{90}$ values is likely due to the lower 
in vitro activity of $\mathbf{3 8}$ against the $P$. berghei parasites versus the $P$. falciparum parasites. It was shown that the compound was active against all asexual erythrocytic stages of $P$. falciparum (i.e. rings, trophozoites, schizonts) and blocked gamete formation (gametocytocidal effect), which very likely explains the observed transmission-blocking effect of $\mathbf{3 8}$. The compound neither exhibited CNS side effects nor cardiovascular side effects and no genotoxicity nor local gastrointestinal toxicity.

In Phase I clinical trials, $\mathbf{3 8}$ was administered as single doses of $10,50,200$ and $500 \mathrm{mg}$ to healthy male subjects to assess safety, tolerability and pharmacokinetics. ${ }^{[12]}$ The effect of food on the PK parameters was also studied in the 200 and $500 \mathrm{mg}$ dose groups. The compound was well tolerated across the whole dose range. Under fed conditions, the AUC (1408 $\mathrm{ng} * \mathrm{~h} / \mathrm{ml})$ was approximately 13 times higher than under fasted conditions. The drug's half-life of approximately $34 \mathrm{~h}$ was not affected by food whereas Tmax was $3.5 \mathrm{~h}$ under fed conditions and only 2 $\mathrm{h}$ under fasted conditions. Compound $\mathbf{3 8}$ has then been tested under fed conditions at a dose of $500 \mathrm{mg}$ in an experimental human malaria infection model, in a clinical trial developed by Medicines for Malaria Venture (MMV) and QIMR Berghofer Medical Research Institute in Brisbane, Australia. ${ }^{[13]}$ With this experiment, the rapid onset of action of $\mathbf{3 8}$ as well as its gametocytocidal effect could be confirmed. To clarify the compound's potential to function as an artemisinin surrogate in ACTs, further investigations are needed. Even though a food effect has been detected and the doses of $\mathbf{3 8}$ required for full efficacy are at the high end, the compound remains interesting due to its mode of action distinct from the peroxide antimalarial drugs. It might therefore be useful in overcoming the resistance issues recently observed with these drugs. ${ }^{[2]}$

\section{Conclusion}

In a collaborative effort between the Swiss TPH and Actelion/ Idorsia Pharmaceuticals Ltd we were able to identify attractive starting points in a phenotypic antimalarial assay which we optimized over the years and finally culminated in the selection of ACT-451840 (38) as a compound for further development. In preclinical investigations we could show cure of $P$. berghei infected mice and an excellent outcome in a humanized SCID mouse model of $P$. falciparum. Further investigations confirmed that the compound acts via a novel mechanism of action ${ }^{[14,15]}$ and showed favourable fast onset of action. Broad in vitro and in vivo characterization resulted in activity over a broad range of malaria parasite strains, favourable behaviour with respect to cross resistance and a potential for blocking transmission among other positive aspects. ${ }^{[10]}$ In phase 1 clinical studies, the compound showed good safety and tolerability, a rather short Tmax and a reasonable $t_{1 / 2}$. Unfortunately, a rather strong food effect was identified, resulting in more than 10 -fold higher exposure in the fed state as compared to the fasted state. A similar food effect has been seen with other lipophilic antimalarial drugs, e.g. lumefantrine. ${ }^{[16]}$ Compound $\mathbf{3 8}$ was finally investigated in an experimentally induced blood stage malaria clinical trial to characterize its antimalarial activity. The compound showed clinical efficacy against $P$. falciparum infections. The PK/PD model enabled prediction of therapeutic effects with cure rates equivalent to artesunate monotherapy, with seven daily doses.[13] Our exciting journey to ACT-451840 is an impressive example of what can be achieved in an industry-academia collaboration in the field of neglected diseases. Malaria is a highly complex disease as the parasite life cycle is partially in humans and partially in the Anopheles mosquito. Drug discovery and development for malaria needs the academic expertise in parasite biology, the access to clinical studies via the MMV and can profit from the industrial expertise in lead optimization toward clinical candidates.

\section{Acknowledgements}

We gratefully acknowledge the numerous contributions from colleagues from Actelion Pharmaceuticals Ltd (till June 2017) and Idorsia Pharmaceuticals Ltd (from July 2017 onward) and from the Swiss TPH to this project which finally resulted in ACT-451840 undergoing clinical phase 2 development.

Received: July 6, 2021

[1] World malaria report 2020: 20 years of global progress and challenges. Geneva: World Health Organization; 2020. Licence: CC BY-NC-SA 3.0 IGO.

[2] C. Boss, S. Wittlin, Med. Chem. Rev. 2018, 53, 293.

[3] R.Hooft vanHuijsduijnen, T.Wells, M.Tanner, S. Wittlin, Malar.J. 2019, 18,94, https://doi.org/10.1186/s12936-019-2728-8.

[4] C. Boss, H. Aissaoui, N. Amaral, A. Bauer, S. Bazire, C. Binkert, R. Brun, C. Bürki, C.-L. Ciana, O. Corminboeuf, S. Delahaye, C. Dollinger, C. Fischli, W. Fischli, A. Flock, M. C. Frantz, M. Girault, C. Grisostomi, A. Friedli, B. Heidmann, C. Hinder, G. Jacob, A. Le Bihan, S. Malrieu, S. Mamzed, A. Merot, S. Meyer, S. Peixoto, N. Petit, R. Siegrist, J. Trollux, T. Weller, S. Wittlin, ChemMedChem 2016, 11, 1995 , https://doi.org/10.1002/cmdc.201600298.

[5] a) H. Aissaoui, C. Boss, O. Corminboeuf, M. C. Frantz, C. Grisostomi (Actelion Pharmacueticals Ltd, Switzerland) WO2010058353 A1, 2010; b) H. Aissaoui, C. Boss, O. Corminboeuf, B. Heidmann, R. Siegrist (Actelion Pharamceuticals Ltd, Switzerland) WO2011083413 A1, 2011; c) H. Aissaoui, C. Boss, O. Corminboeuf, M. C. Frantz (Actelion Pharmaceuticals Ltd, Switzerland) WO 2009141782 A1, 2009.

[6] W. Trager, J. B. Jensen, Science 1976, 193, 673, https://doi.org/10.1126/science.781840.

[7] R. E. Desjardins, C. J. Canfield, J. D. Haynes, J. D. Chulay, Antimicrob. Agents Chemother. 1979, 16, 710, https://doi.org/10.1128/aac.16.6.710.

[8] C. Snyder, J. Chollet, J. Santo-Tomas, C. Scheurer, S. Wittlin, Exp. Parasitol. 2007, 115, 296, https://doi.org/10.1016/j.exppara.2006.09.016.

[9] C.A. Lipinski, F. Lombardo, B. W. Dominy, P. J. Feeney Adv. Drug Deliv. Rev. 1997, 23, 3, https://doi.org/10.1016/s0169-409x(00)00129-0.

[10] A. Le Bihan, R. de Kanter, I. Angulo-Barturen, C. Binkert, C. Boss, R. Brun, R. Brunner, S. Buchmann, J. Burrows, K. J. Dechering, M. Delves, S. Ewerling, S. Ferrer, C. Fischli, F. J. Gamo-Benito, N. F. Gnadig, B. Heidmann, M. B. Jimenez-Diaz, D. Leroy, M. S. Martinez, S. Meyer, J. J. Moehrle, C. L. Ng, R. Noviyanti, A. Ruecker, L. M. Sanz, R. W. Sauerwein, C. Scheurer, S. Schleiferboeck, R. Sinden, C. Snyder, J. Straimer, G. Wirjanata, J. Marfurt, R. N. Price, T. Weller, W. Fischli, D. A. Fidock, M. Clozel, S. Wittlin, PLoS Med 2016, 13, e1002138, https://doi.org/10.1371/journal.pmed.1002138.

[11] I. Angulo-Barturen, M. B. Jimenez-Diaz, T. Mulet, J. Rullas, E. Herreros, S. Ferrer, E. Jimenez, A. Mendoza, J. Regadera, P. J. Rosenthal, I. Bathurst, D. L. Pompliano, F. Gomez de las Heras, D. Gargallo-Viola, PLoS One 2008, 3 , e2252, https://doi.org/10.1371/journal.pone.0002252.

[12] S. Bruderer, N. Hurst, R. de Kanter, T. Miraval, T. Pfeifer, Y. Donazzolo, J. Dingemanse, Antimicrob. Agents Chemother. 2015, 59, 935, https://doi.org/10.1128/AAC.04125-14.

[13] A. Krause, J. Dingemanse, A. Mathis, L. Marquart, J. J. Mohrle, J. S. McCarthy, Br. J. Clin. Pharmacol. 2016, 82, 412, https://doi.org/10.1111/bcp.12962.

[14 R. Brunner, C. L. Ng, H. Aissaoui, M. H. Akabas, C. Boss, R. Brun, P. S. Callaghan, O. Corminboeuf, D. A. Fidock, I. J. Frame, B. Heidmann, A. Le Bihan, P. Jeno, C. Mattheis, S. Moes, I. B. Muller, M. Paguio, P. D. Roepe, R. Siegrist, T. Voss, R. W. Welford, S. Wittlin, C. Binkert, J. Biol. Chem. 2013, 288, 22576, https://doi.org/10.1074/jbc.M113.453159.

[15] R. Brunner, H. Aissaoui, C. Boss, Z. Bozdech, R. Brun, O. Corminboeuf, S. Delahaye, C. Fischli, B. Heidmann, M. Kaiser, J. Kamber, S. Meyer, P. Papastogiannidis, R. Siegrist, T. Voss, R. Welford, S. Wittlin, C. Binkert, J. Infect. Dis. 2012, 206, 735, https://doi.org/10.1093/infdis/jis418.

[16] E. A. Ashley, K. Stepniewska, N. Lindegardh, R. McGready, A. Annerberg, R. Hutagalung, T. Singtoroj, G. Hla, A. Brockman, S. Proux, J. Wilahphaingern, P. Singhasivanon, N. J. White, F. Nosten, Trop. Med. Int. Health 2007, 12, 201, https://doi.org/10.1111/j.1365-3156.2006.01785.x.

\section{License and Terms}

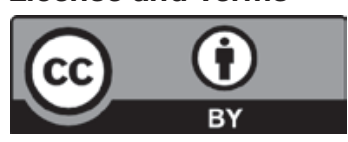

This is an Open Access article under the terms of the Creative Commons Attribution License CC BY 4.0. The material may not be used for commercial purposes.

The license is subject to the CHIMIA terms and conditions: (http:// chimia.ch/component/sppagebuilder/?view=page \&id=12).

The definitive version of this article is the electronic one that can be found at https://doi.org/10.2533/chimia.2021.916 